\title{
Comunicación transgresora de cambio social: epistemologías performativas y eficacia cultural
}

\author{
Transgressive communication for social change: \\ Performative epistemologies and cultural efficacy
}

\author{
Eloísa Nos-Aldás (D) http://orcid.org/0000-0002-8871-976X \\ Universitat Jaume I, España, aldas@uji.es
}

Alessandra Farné $\mathbb{D}$ http://orcid.org/0000-0002-5431-9143

Universitat Jaume I, España, farne@uji.es

\begin{abstract}
This article addresses the concept of cultural efficacy as a paradigm of Communication for Peace and Social Change, heir of the epistemological turn developed by Vicent Martínez Guzmán's Philosophy for Peace. This paper reviews his legacy of peace research as a proposal of dialogic, committed, transformative and interdisciplinary social sciences that define the role of communication in the social fabric from discursive-performative and cultural conceptual and methodological approaches. Finally, we apply this theoretical review of communicative responsibility to a first exploratory study on the refugee and migration crisis.
\end{abstract}

Key words: communication, efficacy, epistemologies, information literacy, social change.

Resumen: Este artículo aborda el concepto de eficacia cultural como paradigma de una Comunicación para la Paz y el Cambio Social, heredero del giro epistemológico desarrollado por la Filosofía para hacer las paces de Vicent Martínez Guzmán. Este trabajo recupera su legado desde las investigaciones para la paz como propuesta de unas ciencias sociales dialógicas, comprometidas, transformadoras e interdisciplinares, que definen el papel de la comunicación en la configuración social desde enfoques conceptuales y metodológicos discursivo-performativos y culturales. Finalmente, aplicamos esta revisión teórica de la responsabilidad comunicativa a un primer estudio exploratorio vinculado con migraciones y refugio.

Palabras clave: cambio social, competencia informacional, comunicación, eficacia, epistemologías. 


\section{Introducción $^{1}$}

Este artículo aborda el concepto de "eficacia cultural" como paradigma de una Comunicación para la Paz y el Cambio Social, heredero del giro epistemológico desarrollado por la Filosofía para hacer las paces de Vicent Martínez Guzmán (La Vall d'Uixó, 1949-Barcelona, 2018). Para ello se revisa como marco teórico-conceptual la investigación para la paz de este autor y su aplicación en la trayectoria de más de 20 años. Esta incluye la transferencia de la línea de trabajo en Comunicación de la Cátedra Unesco de Filosofía para la Paz y del Instituto Interuniversitario en Desarrollo Social y Paz de la Universitat Jaume I, que él mismo constituyó y donde promovió un Máster y un Doctorado en Estudios Internacionales de Paz, Conflictos y Desarrollo (Nos Aldás, 2010a).

Las propuestas de este académico activista -que sintetiza bien Forastelli (2013) - han contribuido, desde su formación en filosofía del lenguaje, a la redefinición y fundamentación del ámbito científico y práctico de las ciencias humanas y sociales como compromiso público, ya que la utilidad pública de la filosofía y la epistemología siempre guiaron su obra.

Este artículo pone a dialogar el legado de Martínez Guzmán -sus propuestas dialógicas, interculturales e interdisciplinares, que ofrecen claves teóricas, metodológicas y prácticas para abordar el estudio de los conflictos humanos y las capacidades que tenemos para transformarlos por medios pacíficos- con la Comunicación del Cambio Social (Nos Aldás y Santolino, 2015). Esta última es el resultado de la evolución de los debates y trabajos en torno a la tradición de la Comunicación para el Desarrollo y para el Cambio Social (CDCS) (Tufte, 2015) desde sus aproximaciones transformadoras de fuerte influencia latinoamericana (Chaparro, 2015; Marí Sáez, 2018) y la Comunicación para la Paz, la Cooperación y la Transformación de Conflictos (Farné y López Ferrández, 2016; Nos Aldás, 2013).

El enfoque de partida es una "filosofía discursiva" basada en una ética discursiva que apela al poder comunicativo de Arendt (1998) y Habermas (1984) (Martínez Guzmán, 2001: 114-116). Martínez Guzmán nos propone "interpelarnos los unos a los otros sobre cómo nos hacemos las cosas y, a su vez, interpelar las estructuras sociales (y los discursos como su representación) sin darlas por hecho, sino aludiendo a la posibilidad de cambiarlas" (Nos Aldás, 2002: 316). Su estudio de las relaciones entre discurso (comu-

\footnotetext{
1 Este trabajo es resultado del proyecto "Comunicación para el Cambio Social y Educación mediática frente a los discursos del odio sobre género e inmigración: análisis de los discursos públicos en el periodo 2016-2019" (UJI-B2019-13). Periodo: 2020-2022.
} 
nicación), culturas y poder, de la performatividad del lenguaje, desvela sus consecuencias políticas y propone un posicionamiento pragmático que asume que podemos hacer las paces (Martínez Guzmán, 2005b), consigna que ha formado parte de las estrategias comunicativas que los movimientos sociales pacíficos llevan décadas demostrando que motivan y movilizan a la ciudadanía. Este libro, subtitulado además "Reflexiones éticas tras el 11-S y el 11-M", recoge el posicionamiento de que "siempre, tras cualquier circunstancia, una y otra vez" debemos volver a intentar trabajar para una convivencia pacífica.

Hablamos de una comprensión del trabajo científico y de sus epistemologías desde la responsabilidad política de incidir en la esfera pública, desde su carácter de espacios donde "actuar juntos [...] 'compartir palabras y actos"” y ejercer el poder "concertadamente", entre todos y todas (Arendt, 1998 y 2005, en Martínez Guzmán, 2015a: 1271) a través de la investigación, la educación y la comunicación.

Este enfoque de "epistemologías para hacer las paces" parte de la comprensión de las desigualdades y la persecución de la justicia desde la libertad y las capacidades (Sen, en Comins y Martínez Guzmán, 2010; Nussbaum, en Giri, 2006), en diálogo con miradas plurales, transgresoras y subalternas como el ecofeminismo (Mies y Shiva, 1997, en Martínez Guzmán, 2001; Puleo, 2011), que sugieren políticas (migratorias, pero también educativas) para culturas basadas en una hospitalidad cosmopolita (Martínez Guzmán, 2003b), enraizadas en el carácter profundamente poscolonial, feminista e intercultural del pensamiento de Martínez Guzmán. Siempre estudió, citó y aprendió de la mirada de mujeres de todas las condiciones y lugares para poner a prueba y completar sus enseñanzas y aprendizajes que, hoy en día, sirven de punto de partida para tesis doctorales en investigaciones para la paz feministas (Castillo Mateu, 2019).

En resumen, y en diálogo con lo que una de las principales discípulas de Martínez Guzmán plantea como los “tres retos epistemológico-normativos que deberían delinear y orientar la investigación para la paz" (Comins Mingol, 2018), este artículo propone el concepto de "eficacia cultural" (Nos Aldás, 2007 y 2010b), a través de la revisión de la epistemología de las ciencias de la comunicación, desde el prisma de un giro epistemológico orientado hacia la educación para la paz, la intervención social y el activismo -lo que en el contexto universitario definiríamos también como transferencia-. Este planteamiento implica incorporar enfoques críticos, propositivos y transformadores, además de otras formas de hacer que generen "marcos teóricos críticos y movilizadores" (Comins Mingol, 2018: 147). 


\section{Interpelaciones del marco teórico y reconstrucción del estado de la cuestión}

Ningún ámbito como el de las ciencias de la comunicación se ve tan interpelado por las ideas que venimos planteando en los párrafos anteriores por su propia razón de ser: estudiar, interpretar, evaluar y diseñar los escenarios de nuestras mediaciones (Martín Barbero, 1998; 1987). Los elementos simbólicos y culturales desempeñan un papel fundamental en la configuración de las sociedades. Entendemos las ciencias de la comunicación aquí desde la llamada comunicología de la liberación y sus enfoques emancipadores (Marí Sáez, 2018), como forma de pensar la comunicación desde la trama de cultura que se articula por medio de procesos de comunicación, prácticas culturales y movimientos sociales, con el objetivo de superar dicotomías y dibujar los mapas complejos de los actuales escenarios de comunicación (Martín Barbero, 2014).

Si nos centramos en el papel sociocultural de la comunicación, en la configuración de una justicia social global, su(s) propia(s) epistemología(s) precisan ser "hackeadas" desde el código abierto (Sampedro, 2015), desde la explicitación de sus intenciones y posicionamientos. Estas tendencias tan actuales son las que ya planteó Martínez Guzmán desde su revisión de la filosofía de la ciencia, desde su argumentación de una epistemología discursiva, crítica y reconstructiva que explicite nuestro posicionamiento, elecciones, intenciones y preocupaciones como científicas, educadoras, políticas o gestoras, porque siempre las tenemos.

Para ello, con palabras de Mignolo (2010), abogamos por una "desobediencia epistémica” noviolenta (Nos Aldás y Farné, 2018) hacia “culturas para hacer las paces” (Comins y Martínez Guzmán, 2010: 56), que transformen las violencias desde las concepciones mismas del saber y de sus aplicaciones y consecuencias éticas, complejizando las cosmovisiones que regulan nuestros comportamientos, pues existen tantas formas de concebir la vida (la paz, la política, la economía) como culturas, lógicas y circunstancias seamos capaces de enumerar. Pero no desde una postura

acrítica y pseudoprogresista de cualquier planteamiento simplemente porque viene de esas otras culturas. [...] parte del reconocimiento del valor de ser discutida y puesta en cuestión y no de la atribución relativista del mismo valor a todas las culturas. Ni todo vale, ni todo vale lo mismo; pero no hemos de decidir "unos" qué vale más, a costa de “otros" (Martínez Guzmán, 2001: 287).

Desde estas premisas, revisamos la epistemología, la razón de ser de las ciencias de la comunicación y las prácticas comunicativas, para pensarse desde sus efectos sociales y culturales en las comunidades, en el corto y en el 
largo plazo, desde la colaboración interdisciplinar, en todas sus dimensiones e interconexiones, desde la salud pública hasta la convivencia pacífica, siendo conscientes de cuántos saberes u opciones se han ido quedando fuera. Porque, como recoge Comins Mingol (2018: 153), a raíz de la "inversión epistemológica [de Martínez Guzmán (2000)] es imperativo corregir el desequilibrio representacional de la paz".

Los conceptos de objetividad y eficacia son dos de las piedras angulares en el desarrollo de las teorías de la comunicación. Estos marcan la concepción de modelos (escenarios) de comunicación lineales, verticales y con desequilibrios de poder o, por el contrario, circulares, horizontales y abiertos a la negociación. El giro epistemológico de Martínez Guzmán reinterpreta esos dos conceptos clave (objetividad y eficacia) desde la intersubjetividad, la solidaridad comunicativa y la interpelación mutua.

Plantea sustituir objetividad por intersubjetividad (Martínez Guzmán, 2005b), porque lo más riguroso, lo que más nos acerca a una realidad, es incorporar tantas perspectivas como sean posibles, construir narrativas integradoras, que incorporen voces con diferentes grados de poder y estilos de saber (el militar y el civil, el científico y el agricultor), y en diálogo (dialogismo). Se trata de una interpelación mutua como parte de la delimitación misma de la comunicación, su estudio y su diseño: concibiendo la comunicación como un proceso de interacción, como una conversación que se autoevalúa y reconstruye constantemente de manera colectiva.

Castillo Mateu (2019) recuerda que Martínez Guzmán (2001: 71) planteó, basándose también en análisis feministas, cómo la violencia cultural (Galtung, 2003) y simbólica (Bourdieu, 2000) puede "hacer opaca nuestra responsabilidad moral" y cómo "hay formas de dominación para las que somos 'ciegos' precisamente por la manera sutil en que penetran las relaciones humanas", lo cual apela incluso a una "crítica de las ciencias modernas como fuente de violencia cultural". Es más, "la objetividad deja al otro fuera de la comunidad moral” (Martínez Guzmán, 2001: 164, en Castillo Mateu, 2019: 48).

Las teorías de la comunicación y sus enfoques científicos actúan como marcos interpretativos, tal y como explican las teorías del framing (Entman, 2007; Goffman, 1974; Lakoff, 2004) sobre todo tipo de discurso, y la ciencia y sus epistemologías son uno más. Es por tanto necesaria una revisión de los mismos y de los posicionamientos políticos y económicos que los dibuja en el seno de la comunidad científica como una comunidad de comunicación internacional. 
Una mirada preñada de memoria histórica que invita a desaprender el modelo de racionalidad occidental desde una racionalidad comunicativa (Apel y Habermas en la segunda generación de la Escuela de Frankfurt) y práctica (Martínez Guzmán 2005a), concibiendo la ciencia como "comunidad discursiva” en la que desaprender lo que hemos dejado de aprender por el camino al no escuchar a los otros y las otras (Marí Sáez, 2012; Martínez Guzmán, 2001: 108; Rodríguez y Moreno, 2016), y con la que transformar los vicios derivados de exponer unos tipos de razón frente a otros (Nos Aldás, 2002: 314). Esas epistemologías del Sur que viene trabajando Sousa Santos (2011), porque la epistemología puede actuar como violencia cultural, o lo contrario.

Esta revisión de la eficacia comunicativa desde los planteamientos de las investigaciones para la paz dialoga con los estudios culturales, los estudios de género, las teorías poscolonialistas y la Comunicación para el Cambio Social (CCS), para definir un paradigma de las ciencias humanas y sociales y del estudio de la comunicación desde una eficacia crítica y transformadora, fundamentado en el análisis de la performatividad de los discursos, de sus implicaciones culturales y las concepciones sociales que promueven o legitiman.

Esta comprensión de las ciencias supera la relación entre sujeto (investigador) y objeto (investigado), para establecer procesos de interpelación y diálogo entre sujetos (grupos, comunidades, personas). Es un enfoque que siempre se planteará el objetivo de comprender y componer la imagen completa de las realidades que se abordan. Si lo trasladamos al campo de la comunicación, hablaríamos de cómo reflejar la complejidad de las experiencias sin dicotomizar, recabando todas las opiniones y experiencias, desde diferentes lugares para evitar excluir o provocar nuevas violencias. Esto, unido a lo anterior, previene los principales riesgos de las representaciones violentas detectadas en la historia de las civilizaciones como la deshumanización, la estigmatización, la desigualdad, la discriminación y la exclusión. Pero, sobre todo, hablamos de una epistemología performativa que contempla la inclusión de valoraciones, que concibe la imposibilidad de ser neutrales en la "ciencia", la investigación o la educación, porque estas precisan reclamar siempre responsabilidades para transformar las injusticias y apuntar a sus causas y causantes; es decir, unas ciencias y su aplicación que "performen", actúen e intervengan proponiendo alternativas.

En conclusión, el giro epistemológico que aquí se aplica concibe los discursos como mediadores entre identidades, creencias, comportamientos y acciones, y propone interpelarlos para interpelarnos desde el convencimiento de la posibilidad de cambiar las condiciones que perpetúan las injusticias, 
por la posibilidad de hacer las cosas de otra manera, y, para ello, de desaprender de forma colectiva lo que nos lo impide.

\section{Redefinición de la eficacia de la comunicación para el cambio social: performatividad, transgresión y eficacia cultural}

La comunicación, como lenguaje, es tanto producto social como práctica social, por lo que estará determinada por el contexto, pero también tendrá consecuencias en el mismo (Fairclough, 1989; Hall, 1997). Partimos de una noción de comunicación como "sistema que organiza la comunicación pública” (Benavides, 1997; Habermas, 1984), donde los individuos, los grupos sociales y las instituciones configuran sus relaciones. Estos escenarios de comunicación son espacios mediados por discursos, donde la interacción entre diferentes interlocutores configura las relaciones e instituciones sociales; configuran las culturas y sociedades que tenemos (Benavides, 1997 y 2011).

En el campo de la comunicación, "eficacia" se ha concebido tradicionalmente como la consecución de los objetivos perseguidos a través de una interacción planificada con unos determinados contextos de recepción y orientada desde unos contextos de producción (De los Ángeles, 1996). Desde la epistemología comprometida con valores que hemos definido, en la que se sustituye la actitud objetiva por la actitud performativa, la comunicación conlleva responsabilidad, por sus implicaciones identitarias, políticas, culturales y sociales. Por tanto, la forma de definir su eficacia y su eficiencia, en cualquier circunstancia, incorpora la valoración de su incidencia simbólica en la configuración de las realidades. Así pues, la CCS tiene como objetivo -y guía para valorar su eficacia- la justicia social y la transformación de las violencias. Sus modelos de comunicación, metodologías de producción de mensajes y, por tanto, de evaluación de resultados, se definirán desde este concepto de eficacia.

En este sentido, hablamos de eficacia “cultural” (Nos Aldás, 2007 y 2010b), porque la CCS necesita incorporar un enfoque integral de los conceptos de violencia cultural y paz cultural (culturas de paz), para desvelar los efectos más sutiles de la comunicación y ser conscientes de sus consecuencias. Galtung (1996) explicita la vinculación entre la violencia cultural, la violencia estructural y la directa, e incorpora al trabajo por la paz (culturas de paz) todos aquellos criterios necesarios para poder definir a las sociedades como pacíficas, más allá de la mera ausencia de guerra.

De esta manera, la responsabilidad de la transformación de las violencias se sitúa también en el plano cultural y simbólico de la construcción y 
legitimación de las ideas, porque los discursos son las semillas de los comportamientos y, por tanto, de las acciones y consecuencias sociales. Así, trabajar la comunicación con eficacia cultural implicará evitar, detectar o transformar cualquier forma de violencia cultural en la producción de discursos de cara a la transformación de los elementos culturales (discursivos y comunicativos) contraproducentes para la equidad y la justicia: "des-legitimar" la violencia y la injusticia, en todas las formas en que estén presentes en los discursos sociales.

Se trata de erradicar la violencia cultural con discursos "alterativos" con criterios de justicia social. El concepto "comunicación alterativa" (Roncagliolo, 1999, citado en Marí Sáez, 2017) alude a la intención de alterar las estructuras que queremos cambiar y expresa esta alteración a través de un juego de palabras, sustituyendo el término "alternativo", que es el habitualmente empleado para este tipo de discursos.

Por tanto, una comunicación que persiga una eficacia cultural anticipará los efectos de la comunicación en los valores, las creencias y los comportamientos sociales. En concreto, en el ámbito de la CCS, se centrará en la influencia de las representaciones (en cuanto elementos culturales y simbólicos) en la transformación estructural de las injusticias y la posible implicación política de las personas (political engagement) y su importancia a largo plazo por encima de apoyos puntuales a determinadas causas.

Se observan aquí las tensiones de la CCS en su doble vertiente planificada y participativa, desde sus procesos estratégicos y su creatividad. La subversión de las epistemologías tradicionales implica, principalmente, el paso de un enfoque persuasivo individual tradicional a uno colectivo, cooperativo y centrado en los procesos, propio de la comunicación comunitaria y participativa (García López, 2010), porque las mismas técnicas y enfoques no son válidos en cualquier contexto.

La comunicación con justicia social tiene una personalidad propia y en coherencia precisa de voces particulares. Voces que aspiren a transgredir las violencias a través de una comunicación con base en un testimonio ético que dialogue con la imaginación moral (Lederach, 2007, en París Albert, 2018), que nos otorga "la capacidad de imaginarnos en una red de relaciones que incluya a nuestros enemigos; la habilidad de alimentar una curiosidad paradójica que abarque la complejidad sin depender de una polaridad dualisítica".

Estos planteamientos transgreden y subvierten lo que se denominan imaginarios reduccionistas de las teorías clásicas de la comunicación (Marí Sáez, 2018), e inciden en la propia concepción de la comunicación, más allá 
de la mera transmisión de información o de su concepción como persuasión. La tradición de estudio de la CCS (Dutta, 2011; Enghel, 2013; Marí Sáez, 2018) redefine su acepción en términos de transformación social a largo plazo e interacción desde las capacidades mutuas, apuntando a la importancia de procesos y relaciones horizontales y dialógicas, por encima de un enfoque instrumental. Esta comunicación se entiende en términos de conversación (como marco alternativo propuesto por Darnton y Kirk, 2011), se exploran retóricas de la invitación (Kaplún, 1998; Foss y Griffin, 1995) por encima de una concepción técnica o instrumental de una comunicación, diseñada desde la opacidad o la imposición.

Por lo tanto, definimos a la CCS desde la eficacia cultural, porque esta persigue objetivos de advocacy, concepto en inglés traducido habitualmente como defensa o incidencia (social o política). Para explicar los matices del término, cabe enfatizar que advocacy incorpora la responsabilidad transversal de la comunicación de los emisores con objetivos colectivos y transformativos a largo plazo de comprender y desarrollar su comunicación, teniendo siempre presente la necesaria combinación y diálogo entre acciones que promuevan una información de calidad, acciones de sensibilización y de educación pública desde una pedagogía política y de cultura de la solidaridad y una constante incidencia hacia los poderes políticos, institucionales, legislativos y mediáticos (Dogra, 2007: 162, basado en Minear, 1987; Cohen 2001: 178).

La perspectiva de "eficacia cultural" se desarrolla, por ende, como herramienta para incorporarla principalmente al proceso de trabajo de los emisores organizacionales que trabajan la comunicación de manera estratégica y planificada. Será necesaria "una teoría estratégica que sepa trabajar con los valores humanos y no sólo con los económicos [...] Que se centre en [...] el largo plazo [...] y las consecuencias culturales". Toda planificación estratégica deberá ser "comunicativa", y pasar "de la mera gestión de la comunicación a la transformación social” (Pérez González, 2003: 86).

La eficacia cultural pretende ampliar la concepción y aplicación de la eficacia comunicativa desde la eficacia social. El concepto de eficacia cultural incide en la responsabilidad política, estructural y a largo plazo de esta comunicación. Esta herramienta teórica ("eficacia cultural") desarrolla una mirada transversal a raíz del concepto de performatividad del lenguaje (Austin, 1976). Trabaja la comunicación como acción que implica unos compromisos en su producción y en su recepción.

La fundamentación de este cambio de perspectiva se refleja en la redefinición del término "persuasión” en los procesos de sensibilización social 
por Martínez Guzmán (2003a: 209). Destaca el "convencimiento" (definición incluida en el Diccionario de uso del Español de María Moliner, 2001) de quien comunica para lograr el convencimiento de aquel a quien se dirige, y destaca el diálogo entre razones y emociones: "sensibilización como persuasión es sensiblemente racional y racionalmente sentimental" (Martínez Guzmán, 2003a: 209) e incorpora una imaginación "deliberativa” que ralentice la interpretación de los interlocutores y les permita tomar sus decisiones más conscientemente, lo cual contrasta con las tendencias de la racionalidad publicitaria extendida en los contextos mediáticos hegemónicos actuales, pero dialoga con la "imaginación moral" (Lederach, 2007), que, a raíz de la constatación, de la relación con la realidad, despierta nuevas actitudes y propuestas en quienes interaccionan.

Estas ideas dialogan con las de Martín Barbero (2003), recogidas por Erro (2010) sobre la "sostenibilidad cultural", aludiendo a que la comunicación con pretensiones educativas - que es la que trabaja para cambiar imaginarios y concepciones- precisará de unos tiempos "pedagógicos y culturales", pues los tiempos del aprendizaje colectivo y de la transformación estructural-cultural son más lentos que los cambios tecnológicos y burocráticos que experimentamos.

Esta idea apunta a la diferencia entre marketing y procesos de construcción cultural (Benavides, 1997: 323), que son procesos relacionales y de construcción de ciudadanía más allá de técnicas de articulación del mercado. Implica una comunicación pública, colectiva, que dinamiza los espacios donde se negocian nuestras identidades y prioridades, la presencia de los diferentes colectivos, su visibilidad.

La eficacia cultural, por tanto, define la comunicación desde su necesaria accountability, como el establecimiento de mecanismos para darnos y pedirnos cuentas (Martínez Guzmán, 2001), para exigir(nos) responsabilidades. Como plantea Oliver (2004) en su estudio sobre el rol de los testimonios, la relación entre interlocutores está sujeta a una "respons(-)[h]abilidad (response-ability) entendida como capacidad de respuesta y responsabilidad en la respuesta” (Gámez Fuentes et al., 2016: 838).

Esta perspectiva incorpora el concepto de "solidaridad comunicativa" a la CCS, tal como la explica el giro epistemológico performativo de Martínez Guzmán (2001: 199-200) desde las teorías de los actos de habla: esforzarnos en hacernos entender y entender lo que nos dicen. Se trata de traducir la solidaridad comunicativa en "sinceridad comunicativa" (Nos Aldás, 2003) y establecer relaciones discursivas horizontales, desde la credibilidad del emisor y la confianza del receptor. 
Estas posturas teórico-organizacionales implican reconocer a los públicos como interlocutores válidos para la transformación de las estructuras de injusticia. En otras palabras, la incorporación a los procesos de análisis y de elaboración de discursos de una perspectiva desde la eficacia cultural exige una sinceridad discursiva que otorgue coherencia respecto a los compromisos establecidos, lo cual conlleva, no solo veracidad, sino también la honestidad del emisor con el receptor, desde la transparencia y la ética.

Estas premisas nos permiten también denunciar y transformar en el terreno de la comunicación comercial tendencias de "apariencia social" que malinterpretan las exigencias sociales sin una coherencia corporativa (como el greenwashing o el pinkwashing, que se refieren a organizaciones que se visten de verde o de rosa solo en pro de beneficios económicos sin un compromiso real y transversal), y mejorar la comunicación en el terreno de sus usos sociales desde un mayor conocimiento de sus consecuencias e influencia.

Por este motivo, cuando hablamos de la cara económica de la comunicación, tanto para emisores comerciales como para campañas sociales que incluyen objetivos de recaudación de fondos, no se pueden obviar las consecuencias culturales. Así, en estos casos, hablamos de buscar una "eficiencia" cultural de la comunicación, que incorpore a sus procesos de búsqueda de beneficios económicos también la evaluación simbólica de sus representaciones, para evitar caer en la legitimación o promoción de modelos sociales y culturales opresivos.

Estas formas de concebir las ciencias desde posturas constructivistas y de paz imperfecta (Muñoz, 2001; Comins Mingol, 2018) nos lleva a hablar de Comunicación del Cambio Social, como aquella que sistematiza a través de procesos iterativos los éxitos que se van produciendo en los diferentes procesos comunicativos cuyo objetivo último es la justicia social, con los que seguir ampliando el conocimiento y la sabiduría cultural compartida para hacer las paces (Nos Aldás et al., 2012).

Lo que Comins Mingol (2018) llama "epistemologías del testimonio", en las cuales revisamos las teorías desde las prácticas, desde la ética y desde sus propios discursos y saberes, buscando lo plural representativo. Además de reivindicar la conexión de la teoría con la praxis, Comins Mingol y Muñoz (2013: 23-58) defienden una filosofía emancipadora que, puesta en diálogo con la Investigación para la Paz, pueda contribuir a construir marcos de sentido capaces de empoderar al ser humano hacia un futuro más justo y pacífico.

Esto dialoga con la propuesta "participante" de Martínez Guzmán que coincide, a su vez, con los enfoques de la CCS (Marí Sáez y Nos Aldás, 2015), 
porque solo es posible profundizar en la comprensión de las situaciones a través del diálogo con sus protagonistas y con quienes tienen experiencia trabajando en ellas. Las teorías solamente pueden confirmarse y completarse de manera iterativa, contrastándolas y comprobándolas con las prácticas, y las prácticas pueden mejorarse con la teoría (Seguí Cosme y Nos Aldás, 2017).

Innovación epistemológica en comunicación transgresora de cambio social: de extranjería a hospitalidad como ejemplo

En el ámbito de las ciencias de la comunicación, el concepto de "eficacia cultural" nos permite plantear una resistencia complejizadora desde el mismo núcleo del proceso de comunicación, puesto que anticipa sus planteamientos y evalúa sus resultados desde su eficacia social. Así, contemplamos la necesidad de la CCS de ser transgresora, basándonos en la aplicación de Mesa et al. (2013: 23) de las ideas de Lagarde y de los Ríos (2000 y 2005). Al ser una comunicación con objetivos de transformación de las estructuras que generan violencias, necesita incorporar la imaginación para transgredir estos marcos establecidos.

Planteamos una comunicación crítica y transformadora en colectivo, concebida "como herramienta de transformación, no mero instrumento de representación” (Nos Aldás, 2002: 318-320). En este último apartado, abordamos el rol del pensamiento crítico, asentado en las epistemologías aquí desarrolladas, en el proceso de aprendizaje (Hooks, 1994) de profesionales de la comunicación a través de la innovación educativa para la paz y su complementariedad con la alfabetización informacional y mediática de toda la ciudadanía (Nos Aldás y Sampedro, 2017; Farné et al., 2018; Nos Aldás, 2019; Nos Aldás et al., 2019), para aprender a comprender y anticipar los compromisos y acciones de las interacciones comunicativas.

Esto se relaciona con la teoría tripartita de la comunicación de Lederach (1995) como expresión, percepción e interpretación, y, si le sumamos la interpelación mutua, que son cuatro elementos en los cuales tenemos que educarnos y profundizar para evitar problemas de comunicación y, en consecuencia, de convivencia, "sin desestimar cada sutil gesto, mirada, silencio, asegurándonos de que comunicamos lo que queremos decir y vemos lo que nos quieren decir" (Martínez Guzmán, 2001: 102-103).

Este modelo plantea la importancia de la anticipación de la performatividad de los discursos, es decir, las intenciones, concepciones, actitudes y valores que cada acto de habla transmite y moviliza. En términos de diversidad, el compromiso de una emisión puede derivar en respeto o en exclusión, 
en reconocimiento o en discriminación. Para procedimentar el análisis de las consecuencias performativas (culturales) de los discursos, delimitamos tres niveles que podemos identificar en todo discurso: 1) las ideas que su creatividad construye (cómo representa las problemáticas, la realidad que comunica); 2) las relaciones que plantea con dichas realidades y los colectivos que las protagonizan (con las personas implicadas); y 3 ) las reacciones (compromisos) que suscita. En otras palabras, la actitud del emisor hacia esa realidad de la que habla; cuáles son los verdaderos objetivos e intenciones de su comunicación y su concepción sobre la comunicación y sus ciompromisos, su verdadera aplicación de la responsabilidad.

Esta aplicación del giro epistemológico en la CCS deriva en una matriz de criterios que nos permiten la producción y la evaluación de discursos públicos desde un marco de eficacia cultural, basado en criterios comunicativos y discursivos desde la mirada performativa (Nos Aldás, 2007; Nos Aldás et al., 2012; Nos Aldás et al., 2015; Pinazo Calatayud y Nos Aldás, 2016), que han demostrado la utilidad y aplicabilidad de estas estrategias comunicativas, no solo para el desarrollo de una comunicación crítica desde los diferentes actores sociales (medios y profesionales de la comunicación, actores gubernamentales y no gubernamentales, movimientos sociales, actores legislativos y educativos) (DevReporter, 2016), sino también para la educación mediática y para la paz con objetivos de ciudadanía crítica global y sujetos políticos inclusivos (Nos Aldás, 2019).

Por tanto, asumimos que los relatos planteados desde una ética del discurso y el "testimonio ético" (Oliver, 2004) anticipan la interacción entre sujetos políticos y la activación de una sensibilidad moral que incrementa su implicación política, tanto en la recepción como en la producción de relatos.

A esto se suma que, en función de la experiencia de la comunicación de los movimientos sociales por una justicia social global, se han demostrado eficaces la transversalidad, la noviolencia, la creatividad colectiva, la sostenibilidad cultural, las emociones movilizadoras como la indignación y la esperanza y los marcos de acción colectiva y justicia social (Castells, 2015), donde las violencias de los marcos hegemónicos asociados a la figura de la víctima sean subvertidas y los enfoques hacia la vulnerabilidad y la resistencia resignificados desde la agencia y la capacidad de transformación de la acción colectiva (Butler et al., 2016). Una acción colectiva que a menudo se sirve de la creatividad colectiva de los movimientos sociales globales por la justicia social - como sistematiza el proyecto de Boyd y Mitchell (2012) y que fundamenta García López (2010)-. 
Otro elemento para abordar las complejidades del sistema de injusticias y desigualdades incluye contemplar una perspectiva de interseccionalidad (Crenshaw, 1991; La Barbera, 2017). Este concepto alude a las intersecciones que se generan a partir los cruces de diferentes discriminaciones (género, etnia, clase, sexualidad, procedencia, entre otros) vinculados al sistema de opresiones y privilegios de los sujetos y los colectivos. Desde un punto de vista comunicativo, la perspectiva interseccional contribuye a comprender las imbricaciones de las desigualdades y así plantear discursos para su transformación, porque "visibilizar las violencias vividas desde los márgenes posibilita explorar narrativas de resistencia que atiendan las necesidades de transformación y re-significación desde diferentes posiciones" (Núñez Puentes et al., 2018: 188).

La traducción pedagógica de este planteamiento de la CCS a criterios de evaluación puede observarse en la Tabla $1 .^{2}$

Nuestras investigaciones demuestran que estos enfoques consiguen una mayor resonancia cultural, es decir, que dialoga y conecta con "consensos culturales establecidos que estén particularmente activos en un determinado clima de opinión" (Nos Aldás et al., 2015), al tiempo que los marcos de protesta, la indignación y las interconexiones de las injusticias y las acciones personales, sociales y políticas incrementan la sensibilidad moral; esto es, siguiendo a la psicología social, la capacidad de un mensaje para despertar en el público un juicio moral que derive en determinadas emociones que conduzcan a la acción (Haidt, 2003, en Pinazo Calatayud y Nos Aldás, 2016: 13).

Retomando los diferentes conceptos y trenzándolos en un modelo de análisis que dialogue también con las propuestas de Strawson (recogidas por Martínez Guzmán, 2001: 183), rastreamos esas epistemologías testimoniales en el modelo desarrollado por Gámez Fuentes y Gómez Nicolau (2017), basado en las posibilidades del "testimonio ético" (Oliver, 2004) que revela en los discursos: la relación entre quien da el testimonio y quien atestigua, el contenido del testimonio, la construcción narrativa de la vulnerabilidad y la resistencia y la vinculación entre la denuncia concreta y el marco general de lucha social donde puede enmarcarse.

Ponemos a dialogar nuestra propuesta de CCS con la actualidad y abordamos un primer estudio exploratorio sobre la crisis de asilo y la urgencia, y carencia, de respuesta (De Andrés et al., 2016). Para ello aplicamos los análisis de la eficacia cultural de la CCS realizados en otros ámbitos como la pobreza, el hambre o el desarrollo (Darnton y Kirk, 2011; Dóchas, 2014; ONGAWA, 2019) a la necesidad de elaborar un marco alternativo que

2 Las tablas 1 y 2 se encuentran en el Anexo, al final del presente artículo (Nota del editor). 
transforme los discursos del odio en otros de hospitalidad y culturas de paz, de modo que la inmigración y la cooperación internacional no se comuniquen como realidades desligadas, ya que están interconectadas desde causas estructurales de violencia y pobreza.

La tradición comunicativa sobre estos temas ha sido, por un lado, vincular la cooperación internacional con marcos de caridad y ayuda -proponiéndose como marcos alternativos la justicia social y la cooperación (Darnton y Kirk, 2011) - y, por otro, abordar la inmigración desde marcos de amenaza, representación del enemigo y el miedo o la identificación de dichos sujetos como "ilegales", entre otros.

En coherencia con las teorías desarrolladas hasta aquí y las preocupaciones por la necesidad de buscar respuestas creativas y contra-narrativas al odio y al racismo (Poole y Giraud, 2019), estas cuestiones precisarían pues de marcos de representación de derechos humanos, justicia social, cosmopolitanismo, interculturalidad y hospitalidad para abordar la solidaridad en un contexto muy mediatizado (Chouliaraki, 2013). Si nos centramos en concreto en los escenarios contemporáneos de crisis de asilo (migraciones desde Siria en busca de refugio, desde el Norte de África hacia España por el Estrecho, por ejemplo), podemos recoger los siguientes marcos tradicionales y sus posibles re-enmarcados (véase Tabla 2).

La CCS se sustentaría en un marco de derechos y, concretamente, en una ciudadanía cosmopolita como derecho público de la humanidad en cuanto sociedad civil global, como derecho a la diferencia y a la interculturalidad, desde el derecho a la interpelación mutua entre culturas y el derecho a la hospitalidad universal (Martínez Guzmán, 1999; y 2001: 287). Por consiguiente, el marco que lo cruza todo son los de la dignidad y la indignación (Martínez Guzmán, 2001; Sampedro, 2015), el de los derechos humanos basados en la dignidad, el desarrollo de los pueblos y las democracias heterogéneas, el derecho a la diferencia y a la interculturalidad, y el de los inmigrantes a la hospitalidad y la interlocución, a ser tomados en cuenta (Martínez Guzmán, 2001: 286 y 291), para lo que este autor propuso ya en 2001 el concepto de hospitalidad frente al de extranjería.

A continuación, ponemos a dialogar esta propuesta de CCS con algunos ejemplos actuales de iniciativas vinculadas a las migraciones y refugio que incorporan marcos alternativos basados en la hospitalidad. Siguiendo el planteamiento del triángulo de violencia y paz de Galtung (1996 y 2003), agrupamos estos ejemplos alrededor de tres categorías.

En primer lugar, para la transformación de la violencia directa encontramos iniciativas de la sociedad civil en el ámbito del socorro humanitario. Se 
trata de acciones como las de los barcos de salvamento marítimos de ONG que rescatan a migrantes que están naufragando en el mar Mediterráneo como, por ejemplo, es el caso de Proactiva-Open Arms, Sea Watch, o SOS Mediterranee-Médicos Sin Fronteras. Estas actividades tienen una repercusión en paliar la violencia directa que sufren los migrantes y que están volcadas a salvarles de la muerte. Este planteamiento de defensa de la vida humana como principio básico se halla también en la comunicación de estas ONG, que adoptan y generan un marco alternativo basado en la cultura de paz, el humanitarismo y la ética del cuidado.

En segundo lugar, para la transformación de la violencia estructural se han implementado varias iniciativas de presión política por parte de la sociedad civil. En este ámbito existe un abanico de diferentes acciones de movilización e incidencia política, orientadas a denunciar las políticas de fronteras, especialmente de la Unión Europea, y las injusticias que suponen para las personas migrantes, a la vez que promueven acciones de acogida e inclusión. Algunos ejemplos de iniciativas con vocación de conseguir cambios políticos y estructurales son Stop Mare Mortum, \#NoUEfortaleza, Refugees Welcome, \#5MAbrazoDeLosPueblos / \#5MEuropeAntiRacist, \#WeAreMore. Desde el punto de vista comunicativo, este tipo de acciones de la sociedad civil asumen y promueven un marco alternativo, con base en la solidaridad, la hospitalidad y la convivencia.

En tercer lugar, para la transformación de la violencia simbólico-cultural, que es la que legitima y sustenta las otras violencias, hemos localizado diferentes acciones en el ámbito de la información y sensibilización. Existen múltiples iniciativas de diversa índole que están enfocadas a transformar los discursos de odio y racismo desde el ámbito de los medios de comunicación tanto convencionales como en las redes sociales. Por ejemplo, existen propuestas centradas en desmentir bulos e informaciones falsas sobre personas extranjeras, como Stop Rumores, BCNAntiRumors o Maldita Migración, algunas enfocadas a transformar el discurso del odio (como \#ElOdioNoEsInocente) y otras orientadas a visibilizar historias positivas de convivencia e interculturalidad como el espacio Desalambre o el especial "La España de los migrantes" de Eldiario.es. Estas acciones comunicativas son heterogéneas, pero en general tienen en común la incorporación y promoción de un marco alternativo basado en valores universales, inclusivos y emancipadores, especialmente los de justicia, diversidad, diálogo e interculturalidad. 


\section{Conclusiones}

Las ideas de este artículo revisan y refuerzan los enfoques de las teorías de la comunicación por medio de un concepto de eficacia cultural, que nos permite incidir en la responsabilidad y las consecuencias culturales de toda comunicación. Específicamente, en diálogo con la epistemología y propuestas de los estudios para la paz, sobre todo las de Martínez Guzmán, profundiza en la violencia cultural y en el análisis de cómo las violencias simbólicas refuerzan la violencia estructural que deriva en violencia directa; en cómo la violencia simbólica, las representaciones, construyen e inciden en nuestras relaciones y acciones.

De aquí hemos extraído una serie de criterios para la transformación pacífica de todos los tipos de violencias en culturas "para hacer las paces" aplicables a, y coincidentes con, los objetivos políticos colectivos de la CCS. Esta propuesta contribuye a una ciencia y una práctica intersubjetiva que incorpora la recuperación de las historias y saberes que han quedado en los márgenes, entre ellos los discursos de paz positiva y paz imperfecta; la narración de los éxitos del cambio social; la visibilización de tantas estructuras y acciones que derivan en el sufrimiento humano.

La eficacia cultural (como interpretación y ampliación del tipo de eficacia comunicativa que precisa la Comunicación para la Paz y del Cambio Social) se ha definido como forma de evaluar, y trabajar desde, la performatividad de los discursos, anticipar y analizar los efectos y compromisos (eficacia social) que toda interacción comunicativa o producción discursiva implica, y cómo pueden crear y reforzar violencias o establecer diálogos activos, reconstruir relaciones, reconocer diferencias e incluirlas. En el caso de la CCS, se trabajan escenarios de comunicación que se preocupan por la justicia social desde lo local a lo global y dialogan con las tendencias más actuales centradas en las capacidades, los compromisos y la interdependencia; la comunicación de denuncia, seguimiento y soluciones; la contextualización, la visibilización, el diálogo con los protagonistas y sus testimonios y la memoria histórica.

En concreto, en este artículo las hemos aplicado en forma de aproximaciones comunicativas para abordar la crisis de refugio y migraciones, resaltando algunos marcos alterativos desde los cuales seguir trabajando transformaciones profundas que cambien la economía de la guerra en economías sociales y solidarias de las que empiezan a existir numerosas prácticas y posibilidades, y de pasar a educarnos y socializarnos en culturas del miedo y la defensa a culturas basadas en la solidaridad y la asunción y conocimiento de nuestras vulnerabilidades, diferencias, conflictos y capacidades, con base 
en valores inclusivos, universales y emancipados (Mesa et al., 2013), preñados de emociones activadoras y cuidados.

Respecto a las limitaciones de esa investigación, el enfoque del monográfico y las restricciones de espacio nos han llevado a dedicar mayor profundidad a la fundamentación del concepto de "eficacia cultural" en los planteamientos epistemológicos de Martínez Guzmán que en el desarrollo procedimental del mismo. Sería deseable seguir observando estas propuestas en las tensiones de los escenarios de comunicación actuales, profundizando en el diálogo entre estas teorías de la transformación pacífica y comunicativa de los conflictos frente a los intereses partidistas y la comprensión hegemónica de la política y el papel de los medios de comunicación masivos y su representación de las migraciones y la crisis de asilo.

Asimismo, es necesario discutir las relaciones paradójicas que surgen entre las actitudes y valores en que se basan estas propuestas, como la idea de comunidad global o la libertad del miedo y de la necesidad de poseer (Darnton y Kirk, 2011), y la cultura hegemónica, y cómo las venimos abordando en la práctica docente con metodologías participativas basadas en la resonancia y la sostenibilidad cultural, en el pensamiento crítico y la educación mediática (Nos Aldás et al., 2019; Nos Aldás, 2019).

\section{Referencias}

Arendt, Hannah (1998), "Sobre la violencia”, en Crisis de la república, España: Taurus. Arendt, Hannah (2005), La condición humana, España: Paidós Ibérica.

Austin, John (1976), How to do things with words, Inglaterra: Oxford University Press.

Benavides, Juan (2011), "La opinión pública y los medios de comunicación social”, en Hortal, Augusto y Etxeberria, Xabier [eds.], Profesionales y vida pública, España: Descleé de Brouwer.

Benavides, Juan (1997), Lenguaje Publicitario. Hacia un estudio del lenguaje en los medios, España: Síntesis.

Bourdieu, Pierre (2000), La Dominación masculina, España: Anagrama.

Boyd, Andrew y Mitchell, Dave [eds.] (2012), Beautiful Trouble. A toolbox for revolution,

Estados Unidos e Inglaterra: OR Books.

Butler, Judit et al. (2016), Vulnerability in Resistance, Inglaterra: Duke University Press.

Castells, Manuel (2015), Redes de indignación y esperanza, España: Alianza.

Castillo Mateu, Laura (2019), Mujer y memoria en las series de prime time de la 1 de TVE (2008-2017): La Señora, 14 de Abril. La República, Isabel y El Ministerio del Tiempo [tesis doctoral], España: Universitat Jaume.

Chaparro Escudero, Manuel (2015), Claves para repensar los medios y el mundo que habitamos: la distopia del desarrollo, Colombia: Desde abajo.

Chouliaraki, Lilie (2013), The ironic spectator: Solidarity in the age of post-humanitarianism, Reino Unido: Polity. 
Comins Mingol, Irene (2018), "Retos epistemológico-normativos de la investigación para la paz”, en Revista de Paz y Conflictos, vol. 11, núm. 2, España: Universidad de Granada.

Comins Mingol, Irene y Martínez Guzmán, Vicent (2010), “Cultura de paz y educación para el desarrollo”, en Burgui, Teresa y Erro, Javier [eds.], Comunicando para la solidaridady la cooperación, España: Foro Comunicación, Educación y Ciudadanía.

Comins Mingol, Irene y Muñoz, Francisco A. (2013), Filosofías y praxis de la paz, España: Icaria.

Crenshaw, Kimberlé (1991), “Mapping the Margins: Intersectionality, Identity Politics, and Violence Against Women of Color”, en Stanford Law Review, vol. 43, núm. 6, Estados Unidos: Stanford University.

Darnton, Andrew y Kirk, Martin (2011), Finding Frames: New ways to engage the UK public in global poverty, Reino Unido: OXFAM-Bond for International Development. Disponible en: http://www.framingmymessage.nl/wp-content/uploads/2013/09/ Finding-Frames-New-ways-to-engage-the-UK-public-in-global-poverty-Bond-2011. pdf [10 de marzo de 2019].

De Andrés, Susana et al. (2016), "La imagen transformadora. El poder de cambio social de una fotografía: la muerte de Aylan”, en Comunicar, vol. 24, núm. 47, España: Universidad de Huelva.

De los Ángeles, Juan (1996), Creatividad Publicitaria. Concepto, estrategias y valoración, España: Ediciones de la Universidad de Navarra (EUNSA).

DevReporter (2016), "Vademécum de información internacional de calidad", en Red Internacional de Comunicadores para el Desarrollo DevReporter. Disponible en: https:// coordinadoraongd.org/wp-content/uploads/2016/07/Vademecum_Devreporter.pdf [12 de marzo de 2019].

Dóchas (2014), The illustrative guide to Dóchas Code of Conduct on Images and Messages. Disponible en: https://dochas.ie/file/illustrative-guide-d\%C3\%B3chas-codeconduct-images-and-messages. [12 de marzo de 2019].

Dogra, Nandita (2007), "Reading NGOs Visually. Implications of visual images for NGO management”, en Journal of International Development, vol. 19, núm. 2, Reino Unido: Wiley.

Dutta, Mohan (2011), Communicating Social Change: Structure, Culture, and Agency, Estados Unidos: Routledge.

Enghel, Florencia (2013), "Communication, Development and Social Change: Future alternatives”, en Wilkins, Karin et al. [eds.], Global Communication: New Agendas in Communication, Inglaterra y Estados Unidos: Routledge.

Entman, Robert (2007), "Framing Bias: Media in the Distribution of Power", en Journal of Communication, vol. 57, núm. 1, Estados Unidos: International Communication Association.

Erro Sala, Javier (2010), “Comunicación, cooperación internacional para el desarrollo y ONGD: un modelo de trabajo desde la educación y la cultura”, en Erro Sala, Javier y Burgui, Teresa [eds.], Comunicando para la Solidaridad y la Cooperación. Cómo salir de la encrucijada, España: Foro de Comunicación, Educación y Ciudadanía.

Fairclough, Norman (1989), Language and Power, Inglaterra: Longman.

Farné, Alessandra et al. (2018), "Communication towards equality for future media professionals: Implementing social awareness through a Cultural Efficacy Festival”, en Congreso Internacional "Trial and Error", Portugal: Universidade do Minho. 
Convergencia Revista de Ciencias Sociales, vol. 27, 2020, Universidad Autónoma del Estado de México

Farné, Alessandra y López Ferrández, Javier (2016), "Comunicación para la Paz: una propuesta pedagógica para el cambio social”, en Opción. Revista de Ciencias Humanas y Sociales, vol. 32, núm. 9, Venezuela: Universidad del Zulia.

Forastelli, Fabricio (2013), "Peace Profile: Vicent Martínez Guzmán”, en Peace Review: A Journal of Social Justice, vol. 25, núm. 3. DOI: 10.1080/10402659.2013.816573 Disponible en: https://doi.org/10.1080/10402659.2013.816573 [15 de marzo de 2019].

Foss, Sonia y Griffin, Cindy (1995), "Beyond Persuasion: A Proposal for an Invitational Rhetoric”, en Communication Monographs, vol. 62, núm. 1, Estados Unidos: National Communication Association.

Galtung, Johan (2003), Paz por medios pacificos. Paz y conflicto, desarrollo y civilización, España: Bakeaz-Gernika Gogoratuz.

Galtung, Johan (1996), “Cultural Violence”, en Peace by Peaceful Means, Inglaterra: Sage.

Gámez Fuentes, María José y Gómez Nicolau, Emma (2017), "El 'testimonio ético' como modelo analítico en la comunicación para el cambio social”, en Actas CIAIQ2017, $6^{\circ}$ Congreso Ibero-americano en investigación cualitativa, España: Ludomedia.

Gámez Fuentes, María José et al. (2016), “Celebrities, violencia de género y derechos de las mujeres: ¿hacia una transformación del marco de reconocimiento?", en Revista Latina de Comunicación Social, núm. 71, España: Universidad de La Laguna.

García López, Marcial (2010), "La comunicación como escenario de empoderamiento ciudadano y para la construcción colectiva de cultura de paz. Pensando otra publicidad social", en Sánchez, Luis y Codorniu, Juan [eds.], Movimiento asociativo y cultura de paz: una mirada desde Andalucía, España: Colección Eirene y Universidad de Granada.

Giri, Ananta (2006), "Cosmopolitanism and Beyond: Towards a Multiverse of Transformations", en Development and Change, vol. 37. DOI: 10.1111/j.14677660.2006.00524.x Disponible en: https://onlinelibrary.wiley.com/doi/10.1111/ j.1467-7660.2006.00524.x [12 de marzo de 2019].

Goffman, Erving (1974), Frame analysis: An essay on the organization of experience, Estados Unidos: Harvard University Press.

Habermas, Jürgen (1984), "El concepto de poder de Hannah Arendt”, en Perfiles Filosóficopoliticos, España: Taurus.

Haidt, Jonathan (2003), “The moral emotions”, en Davidson, Richard et al. [eds.], Handbook of affective sciences, Reino Unido: Oxford University Press.

Hall, Stuart [ed.] (1997), Representation: Cultural Representations and Signifying Practices, Inglaterra: Sage.

Hooks, Bell (1994), Teaching to Transgress. Education as the practice of freedom, Inglaterra: Routledge.

Kaplún, Mario (1998), Pedagogía de la comunicación, España: La Torre.

La Barbera, María Caterina (2017), "Interseccionalidad", en Eunomía, núm. 12, España: Universidad Carlos III.

Lagarde y de los Ríos, Marcela (2005), "Ética y política alternativa”, en Para mis socias de la vida. Claves feministas, España: Horas y Horas.

Lagarde y de los Ríos, Marcela (2000), “Claves feministas para la autoestima”, en Cuadernos Inacabados, núm. 39, España: Horas y Horas.

Lakoff, George (2004), Don't Think of an Elephant: Know Your Values and Frame the Debate, Estados Unidos: Chelsea Green. 
Eloísa Nos-Aldás y Alessandra Farné. Comunicación transgresora de cambio social: epistemologias performativas y eficacia cultural

Lederach, John (2007), La imaginación moral: El arte y el alma de la construcción de la paz, España: Bakeaz.

Marí Sáez, Víctor Manuel (2018), “Análisis de los movimientos-red contemporáneos desde una perspectiva comunicacional y freiriana. Desbordamientos, transformaciones y sujetos colectivos", en Utopia y Praxis Latinoamericana, vol. 23, núm. Extra 3, Venezuela: Universidad del Zulia.

Marí Sáez, Víctor Manuel (2017), “Towards a Critical Political Economy of Indicators. Measuring and Evaluating "Alteratively" Communication, Development and Social Change", en Commons. Revista De Comunicación y Ciudadanía Digital, vol. 6, núm. 1. DOI: 10.25267/COMMONS.2017.v6.i1.01 Disponible en: https://www.researchgate.net/publication/319564897_Editorial_Hacia_una_ Economia_Politica_Critica_del_indicador_Medir_y_evaluar_alterativamente_la_ comunicacion_el_desarrollo_y_el_cambio_social [12 de marzo de 2019].

Marí Sáez, Víctor Manuel (2012), "Building knowledge from the margins: information, knowledge and social movements", en Transinformação, vol. 24, núm. 1. DOI: 10.1590/S0103-37862012000100006 Disponible en: http://www.scielo.br/scielo. php?script=sci_arttext\&pid=S0103-37862012000100006 [12 de marzo de 2019].

Marí Sáez, Víctor Manuel y Nos Aldás, Eloísa (2015), "Prólogo”, en Comunicación para el Cambio Social. La participación y el empoderamiento como base para el desarrollo mundial, España: Icaria.

Martín Barbero, Jesús (2014), “Jesús Martín Barbero: conceptos clave en su obra. Parte 1: Mediaciones", en Pensadores.co [video]. Disponible en: https://www.youtube.com/ watch?v=NveV5ScaZHg [12 de marzo de 2019].

Martín Barbero, Jesús (2003), La educación desde la comunicación, Colombia: Norma.

Martín Barbero, Jesús (1998), "Heredando el futuro: pensar la educación desde la comunicación”, en Cultura y Educación / Culture and Education, vol. 10, núm. 1, España: Taylor and Francis.

Martín Barbero, Jesús (1987), De los medios a las mediaciones: comunicación, cultura y hegemonia, España: Gustavo Gili.

Martínez Guzmán, Vicent (2015a), "Epílogo. Hacer las paces en tiempos de crisis: Veinte años trabajando para hacer las paces en la Universitat Jaume I”, en Nos Aldás, Eloísa et al. [eds.], \#comunicambio: Comunicación y Sociedad Civil para el Cambio Social / \#com4change: Communication and Civil Society for Social Change, España: Fragua.

Martínez Guzmán, Vicent (2005a), "La filosofía para la paz como racionalidad práctica”, en Investigaciones fenomenológicas: Anuario de la Sociedad Española de Fenomenología, núm. 4, España: Universidad Nacional de Educación a Distancia (UNED).

Martínez Guzmán, Vicent (2005b), Podemos hacer las paces. Reflexiones éticas tras el 11-Sy el 11-M, España: Desclée de Brower.

Martínez Guzmán, Vicent (2003a), "Discurso y sensibilización: entre la indignación y la esperanza”, en Benet, Vicente y Nos Aldás, Eloísa [eds.], La publicidad en el Tercer Sector. Tendencias y perspectivas de la comunicación solidaria, España: Icaria.

Martínez Guzmán, Vicent (2003b), "Políticas para la Diversidad: Hospitalidad contra Extranjería”, en Convergencia Revista de Ciencias Sociales, vol. 10, núm. 33, México: Universidad Autónoma del Estado de México.

Martínez Guzmán, Vicent (2001), Filosofía para hacer las paces, España: Icaria. 
Convergencia Revista de Ciencias Sociales, vol. 27, 2020, Universidad Autónoma del Estado de México

Martínez Guzmán, Vicent (2000), "Saber Hacer las Paces. Epistemologías de los Estudios para la Paz”, en Convergencia Revista de Ciencias Sociales, núm. 23, México: Universidad Autónoma del Estado de México.

Martínez Guzmán, Vicent (1999), "Els drets humans, la pau local, la pau global: a manera d'introducció, en Anuari de l'Agrupació Borrianenca de Cultura, núm. 10, España: Agrupació Borrianenca de Cultura.

Mesa, Manuela et al. (2013), Visibles y transgresoras. Narrativas y propuestas visuales para la paz y la igualdad, España: Centro de Educación e Investigación para la Paz (CEIPAZ). Disponible en: http://www.ceipaz.org/visiblesytransgresoras/ [11 de julio de 2018].

Mies, Maria y Shiva, Vandana (1997), Ecofeminismo: teoria, crítica y perspectivas, España: Icaria.

Mignolo, Walter (2010), Desobediencia epistémica. Retórica de la modernidad, lógica de la colonialidad y gramática de la descolonialidad, Argentina: Ediciones del Signo.

Minear, Larry (1987). "The other mission of NGOs: education and advocacy", en World Development, vol. 15, supl. 1, Reino Unido: Elsevier.

Moliner, María (2001), Diccionario de uso del español, España: Gredos.

Muñoz, Francisco (2001), La paz imperfecta, España: Instituto de la paz y los conflictos, Universidad de Granada.

Nos Aldás, Eloísa (2019), “Learning with 'Generation Like’ about digital global citizenship: a case study from Spain”, en Bourn, Douglas [ed.], Bloomsbury Handbook on Global Education and Learning, Inglaterra: Bloomsbury.

Nos Aldás, Eloísa (2013), "Public discourses for nonkilling societes. Peace Research and Communication Trends”, en Evans, Joám [ed.], Nonkilling Media, Estados Unidos: Center for Global Nonkilling, Creighton University.

Nos Aldás, Eloísa (2010a), "La comunicación y los discursos público”, en Investigación para la paz: estudios filosóficos, España: Icaria.

Nos Aldás, Eloísa (2010b), "Comunicación, cultura y educación para la solidaridad y el desarrollo”, en Erro Sala, Javier y Burgui, Teresa [eds.], Comunicando para la Solidaridad y la Cooperación. Cómo salir de la encrucijada, España: Foro de Comunicación, Educación y Ciudadanía.

Nos Aldás, Eloísa (2007), Lenguaje publicitario y discursos solidarios: Eficacia publicitaria, ¿eficacia cultural?, España: Icaria.

Nos Aldás, Eloísa (2003), "Discurso publicitario y sensibilización en las ONGD: de la función social de la publicidad a la responsabilidad de la comunicación social”, en Benet, Vicente y Nos Aldás, Eloísa [eds.], La publicidad en el Tercer Sector: tendencias y perspectivas de la comunicación solidaria, España: Icaria.

Nos Aldás, Eloísa (2002), "Reseña de 'Filosofía para hacer las paces' de Vicent Martínez Guzmán”, en Convergencia Revista de Ciencias Sociales, vol. 9, núm. 27, México: Universidad Autónoma del Estado de México.

Nos Aldás, Eloísa y Farné, Alessandra (2018), "El relato feminista en la cultura popular digital actual: tensiones entre la racionalidad publicitaria y un enfoque político transversal", en Marí Sáez, Víctor y Ceballos Castro, Gonzalo [coords.], Desbordes comunicativos. Comunicación, ciudadania y transformación social, España: Fragua.

Nos Aldás, Eloísa et al. (2019), "Justicia social, culturas de paz y competencias digitales: comunicación para una ciudadanía crítica global en la educación superior”, en Revista Internacional de Educación para la Justicia Social, vol. 8, núm. 1, España: Universidad Autónoma de Madrid. 
Nos Aldás, Eloísa et al. (2012), "La eficacia cultural de la comunicación de las ONGD: los discursos de los movimientos sociales actuales como revisión”, en CIC Cuadernos de Información y Comunicación, vol. 17, España: Universidad Complutense de Madrid.

Nos Aldás, Eloísa y Santolino, Montse (2015), "La Investigación en Comunicación y Cooperación en los nuevos escenarios de movilización social: ONGD, objetivos de justicia social y eficacia cultural", en Revista de la Asociación Española de Investigación de la Comunicación, vol. 2, núm. 4, España: Universidad de Santiago de Compostela.

Nos Aldás, Eloísa et al. (2015), "Evaluación e indicadores de eficacia cultural. Un análisis crítico desde los criterios de éxito de la comunicación actual de los movimientos sociales en España: la resonancia cultural en el caso de la PAH”, en Chaparro, Manuel [ed.], Medios de proximidad: participación social y políticas públicas, España: FraguaCOMandalucía.

Nos Aldás, Eloísa y Sampedro, Víctor (2017), “Educación mediática crítica y participativa: Evolución de la comunicación cultura, nuevas narrativas y dietética digital”, en Actas del III Congreso Internacional de Educación Mediática y Competencia Digital, España: Universidad de Valladolid, Campus Segovia.

Núñez Puente, Sonia et al. (2018), “Comunicación, violencia de género y prácticas de resistencia: narrativas innovadoras para un cambio social”, en Teknokultura, vol. 15, núm. 2, España: Universidad Complutense de Madrid.

Oliver, Kelly (2004), "Witnessing and Testimony", en Parallax, vol. 10, núm. 1, Reino Unido: Taylor and Francis.

ONGAWA (2019), Nadie dijo que fuera fácil. Un relato colectivo, optimista y cuestionador sobre cómo las ONGD implicamos a la ciudadania. Disponible en: https://ongawa.org/ nadiedijoquefuerafacil/ [12 de marzo de 2019].

París Albert, Sonia (2018), "Hacia una reconstrucción de las paces creativas para la ciudadanía global”, en Revista de paz y conflictos, vol. 11, núm. 1, España: Universidad de Granada.

Pérez González, Rafael Alberto (2003), “¿Por qué necesitamos una nueva teoría estratégica?”, en Benavides, Juan y Villagra García, Nuria [eds.], Públicos, instituciones y problemas en la comunicación del nuevo milenio, España: Fundación General de la Universidad Complutense.

Pinazo Calatayud, Daniel y Nos Aldás, Eloísa (2016), “Developing Moral Sensitivity Through Protest Scenarios in International NGDOs' Communication”, en Communication Research, vol. 43, núm. 1. DOI: 10.1177/0093650213490721 Disponible en: https:// journals.sagepub.com/doi/abs/10.1177/0093650213490721 [12 de marzo de 2019].

Poole, Elizabeth y Giraud, Eva (2019), "Right-Wing Populism and Mediated Activism: Creative Responses and Counter-Narratives Special Collection”, en Open Library of Humanities, vol. 5, núm. 1. DOI: 10.16995/olh.438 Disponible en: https://olh. openlibhums.org/articles/10.16995/olh.438/ [14 de marzo de 2019].

Puleo, Alicia H. (2011), Ecofeminismo para otro mundo posible, España: Cátedra.

Rodríguez, Clemencia y Moreno, Martha (2016), "Propuesta para un viraje en el estudio de los medios en Las Márgenes”, en OBETS: Revista de Ciencias Sociales, vol. 11, núm. 1, España: Universidad de Alicante.

Roncagliolo, Rafael (1988), "Las redes de cooperación y la radio comunitaria”, en Chaparro, Manuel [ed.], La democratización de los medios, España: Diputación de Sevilla/EMA-RTV. Sampedro, Víctor (2015), El Cuarto Poder en Red, España: Icaria. 
Seguí Cosme, Salvador y Nos Aldás, Eloísa (2017), "Bases epistemológicas y metodológicas para definir indicadores de eficacia cultural en la comunicación del cambio social", en Commons. Revista de Comunicación y Ciudadanía Digital, vol. 6, núm. 2, España: Universidad de Cádiz.

Sousa Santos, Boaventura de (2011), "Epistemologías del Sur", en Utopia y Praxis Latinoamericana, vol. 16, Venezuela: Universidad del Zulia.

Tufte, Thomas (2015), Comunicación para el cambio social. La participación y el empoderamiento como base para el desarrollo mundial, España: Icaria.

\section{Anexo}

\section{Tabla 1}

\section{Criterios comunicativos y de evaluación de la eficacia cultural}

\begin{tabular}{ll}
\hline Criterio & Descripción \\
\hline 1. Noviolencia & $\begin{array}{l}\text { Evitar cualquier tipo de violencia, incluso simbólica. } \\
\text { Hay que tener cuidado con estereotipos, ridiculizar, } \\
\text { culpabilizar, generalizar, condescendencia, etcétera. }\end{array}$ \\
\hline 2. Reconocimiento & $\begin{array}{l}\text { Reconocer al Otro en igualdad desde la diferencia, } \\
\text { con empatía, relacionarse desde valores universales, } \\
\text { inclusivos y emancipadores. }\end{array}$ \\
\hline 3. Transgresión & $\begin{array}{l}\text { Transformar los marcos de representación hegemónicos } \\
\text { que perpetúan opresiones, promover nuevos imaginarios } \\
\text { a través de la creatividad y la innovación. }\end{array}$ \\
\hline 4. Interseccionalidad & $\begin{array}{l}\text { Contemplar los cruces de las cuestiones de género, } \\
\text { etnia, clase, sexualidad, edad, diversidad funcional, } \\
\text { procedencia, etcétera. }\end{array}$ \\
\hline 5. Inspirac(c)ión & $\begin{array}{l}\text { Fomentar una comunicación propositiva, que ofrezca } \\
\text { modelos de referencia inspiradores de igualdad e invite a } \\
\text { la acción y emancipación. }\end{array}$ \\
\hline
\end{tabular}

Fuente: Elaboración propia. 


\section{Tabla 2}

Principales marcos sobre la crisis de asilo y migraciones

\begin{tabular}{cc}
\hline Marco tradicional & Marco alternativo \\
\hline $\begin{array}{c}\text { Cultura de la defensa } \\
\text { (Economía militar) }\end{array}$ & $\begin{array}{c}\text { Culturas de Paz: noviolencia, } \\
\text { humanitarismo, ética del cuidado } \\
\text { (Economía social y solidaria) }\end{array}$ \\
\hline Cultura del miedo & $\begin{array}{c}\text { Cultura de la solidaridad } \\
\text { (Políticas de fronteras) }\end{array}$ \\
Valores de seguridad, conservación, & convivencia en diversidad $)$ \\
tradición & Valores universales, inclusivos y \\
(Nacionalismo) & emancipadores \\
\hline
\end{tabular}

Fuente: Elaboración propia a partir de Martínez Guzmán (2003) y Mesa et al. (2013).

Eloísa Nos Aldás. Catedrática de la Universitat Jaume I de Castellón (UJI) en el Departamento de Ciencias de la Comunicación, Facultad de Ciencias Humanas y Sociales. Líneas de investigación: comunicación, sociedad civil y cambio social. Publicaciones recientes: Pinazo Calatayud, Daniel, Nos Aldás, Eloísa y Agut Nieto, Sonia (2020), "Comunicar en positivo o negativo en el activismo social”, en Comunicar, núm. 62, España: Universidad de Huelva; Nos Aldás, Eloísa (2019), "From grassroots action to public discourses of cultural peace", en Iranzo, Amador y Farné, Alessandra [coords.] (2019), Comunicación para el cambio social: propuestas para la acción, España: Tirant Lo Blanch; Nos Aldás, Eloísa y Santolino, Montse (2015), "La Investigación en Comunicación y Cooperación en los nuevos escenarios de movilización social: ONGD, objetivos de justicia social y eficacia cultural", en Revista de la Asociación Española de Investigación de la Comunicación, vol. 2, núm. 4, España: Asociación Española de Investigación de la Comunicación. 
Alessandra Farné. Profesora e investigadora del Instituto Interuniversitario de Desarrollo Social y Paz de la Universitat Jaume I de Castellón. Tiene un Máster y Doctorado en Estudios de Paz, Conflictos y Desarrollo, y es licenciada en Relaciones Públicas. Líneas de investigación: comunicación para el cambio social, comunicación de las ONG y movimientos sociales, comunicación online, alfabetización informacional y educación mediática. Publicaciones recientes: Farné, Alessandra y López Ferrández, F. Javier (2016), “Comunicación para la Paz: una propuesta pedagógica para el cambio social”, en Opción. Revista de Ciencias Humanas y Sociales, vol. 32, núm. 9, Venezuela: Universidad del Zulia; Iranzo, Amador y Farné, Alessandra [coords.] (2019), Comunicación para el cambio social: propuestas para la acción, España: Tirant Lo Blanch; Núñez Puente, Sonia, Fernández Romero, Diana y Farné, Alessandra (2018), “Comunicación, violencia de género y prácticas de resistencia: narrativas innovadoras para un cambio social", en Teknokultura, vol. 15, núm. 2, España: Universidad Complutense de Madrid. 